\title{
EDITORIAL
}

DDS - Die Deutsche Schule

113. Jahrgang 2021, Heft 4, S. 373-380

https://doi.org/10.31244/dds.2021.04.01

CC BY-NC-ND 4.0

Waxmann 2021

Julia Hugo, Benjamin Edelstein, Veronika Manitius \& Martin Heinrich

\section{Editorial zum Schwerpunktthema: \\ Chancenungleichheit aus bildungsrechtlicher \\ Perspektive. Inklusion, Übergänge und Homeschooling im Fokus}

\section{Editorial to the Focus Topic: \\ Inequality of Opportunities from an Educational Law Perspective. Inclusion, Transitions, and Homeschooling}

Seit jeher thematisieren und kritisieren Schulforschung, Bildungs- und Erziehungswissenschaft Chancenungleichheiten im deutschen Bildungssystem. Dabei wird jenseits normativer Vorstellungen von Gleichheit und Gerechtigkeit vermehrt auch auf rechtliche Regelungen rekurriert. Wenngleich Bildung und Schule zur Kulturhoheit der Länder zählen (BVerfGE, 1957) und damit landesrechtlich zu regeln sind, fungieren insbesondere Normen mit Verfassungsrang als Bezugspunkte im Diskurs.

So Artikel 3 des Grundgesetzes (GG), dessen Absatz 1 konstatiert: „Alle Menschen sind vor dem Gesetz gleich." Neben diesem formalen, allgemeinen Gleichheitsgrundsatz normiert Absatz 3 in der Folge das Verbot von Diskriminierungen (und Bevorteilungen) aufgrund von Geschlecht, Abstammung, rassistischen Gründen, Sprache, Heimat, Herkunft, Glauben, religiöser Ausrichtung, politischer Weltanschauung (Satz 1) sowie Behinderung (Satz 2). Ferner wird regelmäßig auf Artikel 2 Absatz 1 GG Bezug genommen, der das Recht auf freie Entfaltung der Persönlichkeit garantiert.

Grundrechtlich verbürgte Gleichheitsrechte, Benachteiligungsverbote und Persönlichkeitsrechte legen für juristische Laiinnen - auch in Verbindung mit dem in der Allgemeinen Erklärung der Menschenrechte (Art. 26 AEMR) und in der Kinderrechtskonvention (Art. $28 \mathrm{KRK}$ ) verankerten Recht auf Bildung, das nach Artikel 24 der UN-Behindertenrechtskonvention (UN-BRK) Kindern und Jugendlichen mit sonderpädagogischem Förderbedarf in gleicher Weise zusteht - u.a. den Schluss nahe, es gebe ein von allen Schüler*innen einklagbares gleiches Recht auf Bildung, das „auf 
der Grundlage der Chancengleichheit“ zu verwirklichen sei (Art. 24 Abs. 1 Satz 1 UNBRK).

Abgesehen von der begriffstheoretisch und rechtsphilosophisch voraussetzungsreichen Frage danach, was unter (Chancen-)Gleichheit im Kontext von Bildung zu verstehen ist (vgl. Hugo \& Heinrich, i. Dr.), erweisen sich juristische Auseinandersetzungen mit diesem Themenkomplex in der Regel als differenzierter und die ableitbaren rechtlichen Folgerungen als weniger eindeutig: Zwar bilden die oben genannten Normen ohne Zweifel zentrale Bezugspunkte für rechtliche Argumentationen, z. B. wenn es um den Anspruch auf Nachteilsausgleich geht; doch sind in die rechtswissenschaftliche Analyse stets weitere Aspekte einzubeziehen.

Um nur einige Beispiele zu nennen: Unterschiedliche Rechtsquellen (transnationale Abkommen, Verfassungsrecht, Bundesrecht, Landesrecht etc.) haben unterschiedliche ,Ränge und damit Geltungsbereiche. Hierzu zählt die bereits angesprochene Zuständigkeit der Landesgesetzgebung in Bildungssachen oder die Notwendigkeit, die UNBRK als völkerrechtliche Konvention zunächst in Landesrecht zu transformieren. Ferner ist zu prüfen, ob aus dem objektiv gesetzten Recht ${ }^{1}$ (wie z. B. Art. 24 UN-BRK zum Recht auf inklusive Bildung) überhaupt ein subjektiv einklagbarer Rechtsanspruch des bzw. der Einzelnen (wie der Anspruch eines Kindes mit sonderpädagogischem Förderbedarf auf Zugang zu einer Regelschule) ableitbar ist. Bei gerichtlichen Auseinandersetzungen - gerade im Zusammenhang mit Gleichheitsfragen und Inklusion - ist weiterhin auf den Einzelfall (des jeweiligen Kindes) abzustellen, ohne daraus per se generalisierbare Aussagen (für alle Kinder) ableiten zu können. Außerdem sind gegebenenfalls miteinander kollidierende Grundrechte (z. B. Elternrecht aus Art. 6 Abs. 2 GG vs. staatliche Schulhoheit aus Art. 7 Abs. 1 GG) gegeneinander abzuwägen.

Bisweilen kommt die juristische Argumentation sogar zu - für Rechtslaiinnen - kontraintuitiven Ergebnissen: Eingriffe in Grundrechte (wie die verbindliche Übergangsempfehlung als Eingriff in die Freiheit der Berufswahl aus Art. 12 Abs. 1 GG) sind zwar grundsätzlich nicht verfassungsmäßig; mitunter sind sie aber notwendig und rechtfertigbar, sofern die Grundrechte Dritter tangiert werden und eine VerhältnismäBigkeitsprüfung durch die Rechtsprechung positiv ausfällt. In diesem Fall stuft das Gericht die fragliche Maßnahme (z.B. die Pflicht zum Schulbesuch) als zulässig, geeignet, erforderlich und angemessen im Hinblick auf einen legitimen Zweck ein.

Dem aus Rechtsnormen bzw. ihrer intuitiven Rezeption abgeleiteten Soll-Zustand (wie das oben skizzierte gleiche Recht auf Bildung für alle Schülerinnen) steht ein durch die empirische Forschung beschriebener Ist-Zustand ungleicher Bildungschancen gegenüber, woraus routinemäßig politische Handlungsbedarfe abgeleitet werden.

1 Recht im objektiven Sinne umfasst „die Rechtsordnung, dh (sic) die Gesamtheit der Rechtsvorschriften“ (Weber, 2019, S. 1155). 
Spätestens seit den 1970er Jahren (vgl. Deutscher Bildungsrat, 1970) werden soziale Ungleichheiten im deutschen Bildungssystem fortlaufend kritisiert, analysiert und bildungspolitisch diskutiert. Trotz zahlreicher Reformbestrebungen handelt es sich offenkundig um ein Dauerthema der Erziehungs- und Bildungswissenschaften bzw. einen Dauerbefund der empirischen Bildungsforschung (vgl. Böttcher, 2021).

Chancenungleichheit wird dabei - das liegt in der Natur der Sache - stets mit Blick auf bestimmte Schüler ${ }^{*}$ innengruppen diskutiert, etwa solche mit niedrigem sozioökonomischem Status (vgl. z. B. OECD, 2018), mit Migrationshintergrund oder mit Fluchterfahrung (vgl. z.B. Autorengruppe Bildungsberichterstattung, 2020). Einem weiten Inklusionsbegriff folgend bilden Schüler*innen mit sonderpädagogischem Förderbedarf oder chronischer Krankheit eine weitere relevante Gruppe. Dabei wird das Ausmaß bestehender Ungleichheiten vornehmlich an Indikatoren der Bildungsbeteiligung (etwa gruppenspezifische Partizipationsraten oder Abschlussquoten in verschiedenen Bildungsgängen) und in jüngerer Zeit verstärkt auch an gruppenspezifischen Kompetenzungleichheiten festgemacht, wie sie aus nationalen (z. B. IQB-Bildungstrend, vgl. Stanat, Schipolowski, Mahler, Weirich \& Henschel, 2018) und internationalen Schulleistungsvergleichsstudien (z. B. PISA, TIMSS etc.; vgl. Reiss, Sälzer, Schiepe-Tiska, Klieme \& Köller, 2016) hervortreten. Angestoßen durch die Ratifizierung der UN-BRK und die damit einhergehende Verpflichtung zum Aufbau eines inklusiven Bildungssystems in Deutschland verweisen jüngst statistische Inklusions- und Exklusionsquoten (vgl. z. B. Klemm, 2018) auf die ungleichen Zugangschancen zu einer Regelschule von Kindern und Jugendlichen mit sonderpädagogischem Förderbedarf.

Für die (Re-)Produktion besagter (Chancen-)Ungleichheiten stechen immer wieder diverse Bedingungsfaktoren hervor: So beispielsweise die Selektivität des deutschen Bildungssystems als „Hindernisparcours“ (Helsper \& Kramer, 2007, S. 439), die insbesondere an den Übergangsmodalitäten vom Primar- in den Sekundarbereich sowie am hierarchischen Aufbau der Sekundarstufe festgemacht wird. Hierbei spielen primäre und sekundäre Herkunftseffekte (vgl. Boudon, 1974) ${ }^{2}$, aber auch rechtliche und institutionelle Rahmenbedingungen der Schulorganisation zusammen (vgl. Dumont, Maaz, Neumann \& Becker, 2014).

An die säkulare Kritik der Struktur des deutschen Schulwesens schließt sich die aktuell kurante Frage nach der Legitimation von Förderschulen und anderen separierenden Organisationsformen bei gleichzeitiger Verpflichtung Deutschlands zum Aufbau eines inklusiven Schulsystems an (Art. 24 Abs. 1 i. V.m. Art. 4 Abs. 2 UN-BRK). Ana-

2 Mit dem Konzept der primären Herkunftseffekte werden die im Schnitt schlechteren Schulleistungen von Schüler*innen aus bildungsfernen und damit i.d. R. weniger lernförderlichen Elternhäusern beschrieben; sekundäre Herkunftseffekte beziehen sich demgegenüber auf vom Leistungsstand der Kinder und Jugendlichen unabhängige Unterschiede, etwa das je nach sozialer Zugehörigkeit divergierende Schulwahlverhalten von Eltern (vgl. Boudon, 1974). 
lysen, statistische Kennwerte und Bestandsaufnahmen der letzten zehn Jahre zeugen durchweg von einer defizitären Umsetzung der UN-Konvention sowohl auf rechtlicher wie auf institutioneller Ebene. Dies wird von Erziehungs- und Rechtswissenschaft gleichermaßen moniert (vgl. jüngst Steinmetz, Wrase, Helbig \& Döttinger, 2021).

Aktuell ist die antizipierte Verschärfung sozialer Disparitäten durch den pandemiebedingt eingeschränkten Schulbetrieb und den Distanzunterricht im Elternhaus Gegenstand von Forschung und Diskussion. Wenngleich statistische Analysen verschiedentlich Hinweise auf entsprechende Effekte geben (vgl. z. B. Destatis, WZB \& BiB, 2021), ist die empirische Befundlage zu dieser Frage insgesamt heterogen (vgl. die Übersichtsbeiträge in Fickermann, Edelstein, Gerick \& Racherbäumer, 2021). Das infektionsschutzrechtlich legitimierte Schulbesuchsverbot hat zur Frage nach dem Wesensgehalt der aus dem staatlichen Bildungs- und Erziehungsauftrag (Art. 7 Abs. 1 GG) abgeleiteten Schulpflicht geführt (vgl. Reimer, 2021). Bis dato ging die traditionelle Konzeptualisierung als Schulbesuchspflicht mit einem grundsätzlichen, mithin strafbewehrten Verbot jeglicher Homeschooling-Formate einher (vgl. jüngst Hauck, 2020).

Mit dem exemplarischen Fokus auf Inklusion, Übergänge und Homeschooling nimmt das vorliegende Heft damit erziehungs- und bildungswissenschaftliche Themenfelder in den Blick, die in engem Zusammenhang mit Fragen der Chancenungleichheit stehen und um die darüber hinaus ein breiter juristischer Fachdiskurs existiert (vgl. z. B. Bernhard (2016) sowie Gasterstädt (2019) zu Inklusion; Barczak (2011) zu Übergangsregelungen; von Lucius (2017) zu Homeschooling). Ziel ist es, sich mitunter auftretenden Divergenzen zwischen erziehungswissenschaftlicher Rezeption und rechtswissenschaftlicher Auslegung rechtlicher Regelungen anzunähern und damit den inter- und transdisziplinären Austausch anzuregen. Als Hilfestellung sind die wesentlichen rechtlichen Regelungen, auf die in den nachfolgenden Beiträgen Bezug genommen wird, in einem das Heft begleitenden Online-Anhang zusammengetragen: https://www. waxmann.com/artikelART104608. Ein Link zum entsprechenden Dokument befindet sich im jeweiligen Beitrag zu Beginn des Literaturverzeichnisses.

Der Einstieg in die bildungsrechtliche Materie erfolgt anhand von drei Grundlagenbeiträgen, in denen die im jeweiligen Gegenstandsbereich herrschende Rechtsdogmatik aus völker- und/oder verfassungsrechtlicher Perspektive dargestellt wird.

Der Beitrag von Susanne Kroworsch zum Fokus Inklusion führt in (völker-)rechtliche Verpflichtungen, Herausforderungen und Irrtümer bei der Umsetzung der UN-Behindertenrechtskonvention in Deutschland ein. Hierfür werden Inhalte und Ansprüche der Konvention dem Status quo der Umsetzung einerseits und der in diesem Zusammenhang ergangenen Rechtsprechung andererseits gegenübergestellt. Im Ergebnis leitet die Autorin eine klare Positionierung ab: Inklusive Bildung und damit Chancengleichheit sei nur durch die Abschaffung von Förderschulen zu erreichen. 
Ebenfalls aus verfassungsrechtlicher Perspektive unterzieht der Beitrag zum Fokus Übergänge von Wolfram Cremer und Antonia Tobisch die in den Bundesländern geltenden Übergangsregelungen einer rechtlichen Überprüfung anhand des Maßstabs des Grundgesetzes. Die dezidiert juristische Analyse der Frage, inwiefern die jeweiligen Übergangsregimes verfassungsmäßig sind - oder nicht - bietet den Leser*innen einen Einblick in authentische juristische Deduktion. Die Übergangsregelungen werden zunächst in „Verbindliche“ und „Unverbindliche“ sowie das sogenannte „Beratungsmodell mit Präklusionswirkung“ differenziert. Es zeigt sich, dass nicht zuletzt wegen des Einflusses von Herkunftseffekten auf die Übergangsempfehlung ausschließlich unverbindliche Regelungen verfassungsmäßig sind.

Ausgangspunkt von Franz Reimers Beitrag zum Fokus Homeschooling ist die verfassungsrechtlich begründete Notwendigkeit von Schulpflicht zur Realisierung des staatlichen Bildungs- und Erziehungsauftrags und damit von Chancengleichheit. Vor dem Hintergrund der Corona-Pandemie unternimmt er eine Neubewertung eines bekannten Problemkomplexes: nämlich der Frage, ob Schulpflicht in Schulbesuchspflicht aufgeht. Dabei wird die in Deutschland übliche Gleichung „Schulpflicht = Schulbesuchspflicht“ als „Anomalie“ im europäischen Vergleich ausgewiesen. Das im Rahmen der Pandemie infektionsschutzgesetzlich legitimierte Schulbesuchsverbot einerseits und Homeschoolinggebot andererseits fördern nach Ansicht des Autors eine Verwechslung der bisherigen Rechtsdogmatik zu Tage. Demnach ist Schulbesuchspflicht lediglich Mittel zur Erreichung des übergeordneten Telos von Schulpflicht, nämlich der Bildung und Erziehung von Kindern und Jugendlichen. In den Fällen, in denen sich das Mittel als teleologisch dysfunktional erweist, gelte es von dieser Gleichung abzuweichen.

$\mathrm{Ob}$ die bildungsrechtliche Auseinandersetzung tatsächlich zu einem Abbau von Divergenzen geführt hat bzw. führen kann, diskutiert Wolfgang Böttcher in einem die Grundlagenbeiträge kommentierenden Diskussionsbeitrag, der, nun aus erziehungsund bildungswissenschaftlicher Perspektive, sich ergebende Fragen, Spannungsmomente und Einsichten reflektiert. Er kommt zu folgender Erkenntnis: Wenngleich juristische Argumentationen neue Aspekte zu Tage brächten, so eigneten sie sich nicht dazu, die „Dilemmata der Pädagogik“ aufzulösen. Gleichwohl erscheint ihm ein stärkerer Austausch der beiden Disziplinen, der Rechts- und Erziehungswissenschaft, so unabdingbar wie aussichtsreich.

Die rechtliche Auseinandersetzung mit den im Heft behandelten Themenfeldern wird anschließend anhand von drei fallbezogenen Berichten fortgesetzt, die auf die Beschreibung und Diskussion paradigmatischer Beschlüsse und Urteile der Rechtsprechung fokussieren, die für Lehrkräfte, Schulleitungen und Eltern, aber auch für die Schuladministration von besonderem Interesse sind.

Wolfgang Bott gibt ausgehend von den rechtlichen Auswirkungen der UN-BRK einen Überblick über die Rechtsprechung der Verwaltungsgerichte zu Inklusion und fragt, 
inwiefern die Umsetzung von inklusiver Bildung durch die Judikative gefördert werden kann. Er resümiert, dass die Rechtsprechung Chancengleichheit nur formell auf Verfahrensebene sichern könne. Die Frage nach materieller Chancengleichheit erweise sich demgegenüber als Einzelfallentscheidung.

Die Vielfalt möglicher rechtlicher Übergangsmodalitäten ist Gegenstand des Beitrags von Wolfgang Bott und Heinz Kipp. Zunächst werden im historischen Überblick die Empfehlungspraktiken im Bundesland Hessen dargestellt und rechtlich eingeordnet. Es zeigt sich, dass in fast allen Bundesländern den Eltern das Letztentscheidungsrecht bei der Schulwahl zukommt. Diese Liberalisierung der Übergangsregelung geht mit einer Zunahme der Verantwortung auf Seite der einzelnen, aufnehmenden Schule einher. Daher wird - in Reaktion auf die rechtliche Einordnung - anschließend der Versuch eines pädagogischen Kommentars unternommen. Auf Basis schulpraktischer Erfahrungen unterbreitet Heinz Kipp einen Vorschlag für die pädagogische Begleitung eines gelingenden Übergangsmodus.

In Ergänzung zu Reimers verfassungsrechtlicher Perspektive untersucht der Beitrag von Andreas Vogt konkrete Fälle des Bundesverfassungsgerichts sowie der Familiengerichte zum Thema Homeschooling. Entgegen der gängigen Rechtsprechung argumentiert er, dass Homeschooling nicht per se Kindeswohlgefährdung bedeute. Schulpflicht im Sinne einer Schulbesuchspflicht stelle sich diesbezüglich als unzulässiger Eingriff in die Rechte der Eltern und Kinder dar, der die Umstände des Einzelfalls nicht angemessen berücksichtige.

Das Heft schließt mit einem Werkstattbericht von Marcel Helbig und Rita Nikolai zu einem Projekt, das in eigener Weise auf schulrechtliche Informationsbedürfnisse reagiert: eine durch Auswertung schulrechtlicher Regelungen der 16 Bundesländer aus den Schuljahren 1949/50 bis 2009/10 entstandene Datenbank, in der verschiedenste rechtliche Indikatoren abgerufen werden können (etwa zum Aufbau des Schulwesens, zu Übergangs- und Versetzungsregelungen u.v.m.). Die Autor*innen zeigen Anschlussmöglichkeiten der in der Datenbank aufbereiteten und zur Verfügung gestellten Informationen für Fragen der Erziehungswissenschaft und Bildungsforschung, der Politikwissenschaft, der Rechtsgeschichte und -soziologie auf. Die Datenbank steht damit paradigmatisch für einen gelingenden inter- und transdisziplinären Austausch.

Das Bundesverfassungsgericht hat die Bedeutung der Bildungsforschung für die Bildungspolitik bereits 2002 herausgestrichen: „Seine [des Staates] Aufgabe ist es aber, auf der Grundlage der Ergebnisse der Bildungsforschung bildungspolitische Entscheidungen zu treffen" (BVerfG, 2002). Die bildungsrechtlichen Auseinandersetzungen mit genuin erziehungs- und bildungswissenschaftlichen Themen in diesem Heft zeigen, dass ungleiche Bildungschancen und diesbezügliche empirische Erkenntnisse auf die eine oder andere Weise stets auch Eingang in juristische Analysen finden. Es bleibt zu hoffen, dass das vorliegende Heft Einsichten zutage fördert, die einem konstrukti- 
ven Dialog zwischen Rechts- und Erziehungswissenschaft - nicht nur zu den drängenden Fragen von Inklusion, Übergängen und Homeschooling - förderlich sind.

\section{Literatur und Internetquellen}

Autorengruppe Bildungsberichterstattung. (2020). Bildung in Deutschland 2020. Ein indikatorengestützter Bericht mit einer Analyse zu Bildung in einer digitalisierten Welt. Zugriff am: 21.09.2021. Verfügbar unter: https://www.bildungsbericht.de/de/Bildungsbericht 2020barrierefrei.pdf/view.

Barczak, T. (2011). Der Übergang von der Grundschule in die Sekundarstufe als Grundrechtsproblem. Eine rechtliche Untersuchung unter Berücksichtigung sozialer Herkunftseffekte (Münsterische Beiträge zur Rechtswissenschaft - Neue Folge, Bd. 12). Baden-Baden: Nomos. https://doi.org/10.5771/9783845233291

Bernhard, T. (2016). Anforderungen an ein inklusives Bildungssystem nach der UN-Behindertenrechtskonvention. Eine Untersuchung der Rechtslage im Freistaat Bayern (Schriften zum Bildungs- und Wissenschaftsrecht, Bd. 18). Baden-Baden: Nomos. https://doi. org/10.5771/9783845274553

Böttcher, W. (2021). Chancenungleichheit Oder: Die ewige und langweilige Geschichte der sozialen Selektion im Bildungswesen. In L. Brockmann, C. Hack, A. Pomykaj \& W. Böttcher (Hrsg.), Soziale Ungleichheit im Sozial- und Bildungswesen. Reproduktion und Legitimierung (S. 17-34). Beltz Juventa.

Boudon, R. (1974). Education, Opportunity, and Social Inequality. Changing Prospects in Western Society. New York: Wiley.

BVerfG (Bundesverfassungsgericht). (1957). Urteil vom 26. März 1957 - 2 BvG 1/55.

BVerfG (Bundesverfassungsgericht). (2002). Beschluss vom 16. April 2002 - 1 BvR 279/02 (Rn. 1-37). Zugriff am: 20.09.2021. Verfügbar unter: https://www.bundesverfassungs gericht.de/SharedDocs/Entscheidungen/DE/2002/04/rk20020416_1bvr027902.html

Destatis (Statistisches Bundesamt), WZB (Wissenschaftszentrum Berlin für Sozialforschung) \& BiB (Bundesinstitut für Bevölkerungsforschung). (Hrsg.). (2021). Datenreport 2021. Ein Sozialbericht für die Bundesrepublik Deutschland. Bonn: bpb. Zugriff am: 21.09.2021. Verfügbar unter: https://www.destatis.de/DE/Service/Statistik-Campus/ Datenreport/Downloads/datenreport-2021.pdf?_blob=publicationFile.

Deutscher Bildungsrat. (1970). Strukturplan für das Bildungswesen (2. Aufl.). Klett.

Dumont, H., Maaz, K., Neumann, M., \& Becker, M. (2016). Soziale Ungleichheiten beim Übergang von der Grundschule in die Sekundarstufe I. Theorie, Forschungsstand, Interventions- und Fördermöglichkeiten. Zeitschrift für Erziehungswissenschaft, 17 (24. Suppl.), 141-165. https://doi.org/10.1007/s11618-013-0466-1

Fickermann, D., Edelstein, B., Gerick, J., \& Racherbäumer, K. (Hrsg.). (2021). Schule und Schulpolitik während der Corona-Pandemie: Nichts gelernt? (Die Deutsche Schule, 18. Beiheft). Münster: Waxmann. https://doi.org/10.31244/9783830994589

Gasterstädt, J. (2019). Der Komplexität begegnen und Inklusion steuern. Eine Situationsanalyse zur Umsetzung von Artikel 24 der UN-BRK in Deutschland. Wiesbaden: Springer VS. https://doi.org/10.1007/978-3-658-27838-0

Hauk, J. (2020). Die Pflicht zum Schulbesuch. Zur Frage nach einem Recht auf Homeschooling unter Berücksichtigung grundrechtlicher Freiheiten von Eltern und Schülern (Studien zu Staat, Recht und Verwaltung, Bd. 29). Baden-Baden: Nomos. https://doi.org/10.5771/ 9783748904564

Hugo, J. \& Heinrich, M. (i. Dr.). Chancengleichheit oder Bildungsgerechtigkeit: zwischen formaler Gleichheit und normativer Wertigkeit. Differenzen und Anschlussfähigkei- 
ten der Erziehungswissenschaft an den rechtlichen Diskurs. In W. Böttcher, C. Luig, L. Brockmann \& C. Hack (Hrsg.), Chancenungleichheit: geplant, organisiert, rechtlich kodifiziert. KBBB-Tagungsband (S. 141-152). Münster: Waxmann.

Klemm, K. (2018). Unterwegs zur inklusiven Schule. Lagebericht 2018 aus bildungsstatistischer Perspektive. Gütersloh: Bertelsmann Stiftung. https://doi.org/10.11586/2018050

OECD (Organization for Economic Cooperation and Development). (2018). Equity in Education: Breaking Down Barriers to Social Mobility. Paris: OECD Publishing. https://doi. org/10.1787/9789264073234-en

Reimer, F. (2021). Der verfassungsrechtliche Schulbegriff: Neuer Wein in neue Schläuche! Recht der Jugend und des Bildungswesens, 69 (1), 58-77. https://doi.org/10.5771/00341312-2021-1-58

Reiss, K. Sälzer, C., Schiepe-Tiska, A., Klieme, E., \& Köller, O. (Hrsg.). (2016). PISA 2015. Eine Studie zwischen Kontinuität und Innovation. Münster: Waxmann.

Stanat, P., Schipolowski, S., Mahler, N., Weirich, S., \& Henschel, S. (Hrsg.). (2018). IQB-Bildungstrend 2018. Mathematische und naturwissenschaftliche Kompetenzen am Ende der Sekundarstufe I im zweiten Ländervergleich. Münster: Waxmann.

Steinmetz, S., Wrase, M., Helbig, M., \& Döttinger, I. (2021). Die Umsetzung schulischer Inklusion nach der UN-Behindertenrechtskonvention in den deutschen Bundesländern (Recht und Gesellschaft - Law and Society, Bd. 15). Baden-Baden: Nomos. https://doi. org/10.5771/9783748924401-1

von Lucius, J. (2017). Homeschooling: Eine verfassungsrechtliche Untersuchung des Rechts der Eltern, ihre Kinder zu Hause zu unterrichten (Gesetzgebung und Verfassung, Bd. 9). Baden-Baden: Nomos. https://doi.org/10.5771/9783845272252

Weber, K. (2019). Recht. In C. Creifelds (†) \& K. Weber (Hrsg.), Rechtswörterbuch (23., neu bearb. Aufl., S. 1155). München: C. H. Beck. 